\title{
Grain Selection and Texture Evolution in Directionally Solidified Al-Zn Alloys
}

\author{
FRÉDÉRIC GONZALES and MICHEL RAPPAZ
}

In a recent contribution, we have reported that dendrites in the aluminum-zinc binary system can change their growth direction continuously from $\langle 100\rangle$ to $\langle 110\rangle$ as the concentration of zinc $C_{\mathrm{Zn}}$ increases from 5 to $90 \mathrm{wt}$ pct. ${ }^{[1]}$ This was interpreted as an influence of the hcp $\mathrm{Zn}$ element on the low anisotropy of the solid-liquid interfacial energy of aluminum. Seaweed morphologies were even observed at the start and end of this so-called "dendrite orientation transition" (DOT), i.e., for $C_{\mathrm{Zn}} \approx 30$ and $55 \mathrm{wt}$ pct. In the present work, we analyze how this DOT influences the selection of grains and the evolution of the texture in directionally solidified (DS) specimens. The solidification texturing of $\langle h k 0\rangle$ dendritic specimens is shown to be similar to that occurring in normal $\langle 100\rangle$ specimens. It is analyzed on a similar basis by considering a random orientation distribution of nuclei at the surface of the chill plate and a minimum undercooling criterion. This produces $\langle h k 0\rangle$ textures with grains that look fairly "equiaxed" in transverse sections. For seaweed morphologies, which also exhibit an $\langle h k 0\rangle$ texture parallel to the thermal gradient, the grain selection is slower and the grains appear more elongated in transverse sections. Their elongation occurs along a (001) plane, i.e., along a $\langle 1 \overline{2} 0\rangle$ direction for $\langle 210\rangle$ seaweeds $\left(C_{\mathrm{Zn}}=30 \mathrm{wt}\right.$ pct $)$ and along a $\langle 1 \overline{1} 0\rangle$ direction for $\langle 110\rangle$ seaweeds $\left(C_{\mathrm{Zn}}=55 \mathrm{wt} \mathrm{pct}\right)$. The scanning electron microscopy (SEM) observations reveal that this elongation is accompanied by a microsegregation pattern that is mainly parallel to (001) planes. This indicates that seaweeds at the onset and end of the DOT grow with some type of layered structures, but their detailed growth and grain selection mechanisms are still unknown.

DOI: $10.1007 / \mathrm{s} 11661-008-9546-\mathrm{x}$

(C) The Minerals, Metals \& Materials Society and ASM International 2008

\section{INTRODUCTION}

AluminUM-ZINC alloys, which constitute the basis of the 7000 series alloys, appear to be also very interesting from a fundamental point of view of solidification. Indeed, in a recent contribution, ${ }^{[1]}$ we have shown that $\mathrm{Al}-\mathrm{Zn}$ dendrites produced by directional or Bridgman solidification do not necessarily grow along the usual $\langle 100\rangle$ directions characteristic of cubic metals. ${ }^{[2]}$ Below $25 \mathrm{wt}$ pct of zinc, fcc aluminum dendrites do grow along $\langle 100\rangle$, but beyond $60 \mathrm{wt}$ pet, their growth direction was clearly identified as $\langle 110\rangle$ by electron backscattered diffraction (EBSD). In between these two concentrations, a gradual dendrite orientation transition (DOT) from $\langle 100\rangle$ to $\langle 110\rangle$ occurs in a (001) plane as the zinc concentration $C_{\mathrm{Zn}}$ increases. At the start and end of this DOT, i.e., for $C_{\mathrm{Zn}} \approx 30$ and $55 \mathrm{wt}$ pct, textured seaweeds were observed instead of dendrites.

The change of dendrite growth direction is not new for organic alloys or salty solutions. ${ }^{[3,4]}$ For example, Akamatsu et al. ${ }^{[3]}$ have observed in situ the various growth morphologies in the organic transparent $\mathrm{CBr}_{4}-\mathrm{C}_{2} \mathrm{Cl}_{6}$ system confined in between two glass plates, i.e., nearly two-dimensional (2-D) growth. When the

FRÉDÉRIC GONZALES, Postdoctoral Student, and MICHEL RAPPAZ, Professor, are with the Computational Materials Laboratory, Ecole Polytechnique Fédérale de Lausanne, Lausanne, CH-1015, Switzerland. Contact e-mail: michel.rappaz@epfl.ch

Manuscript submitted November 12, 2007.

Article published online May 22, 2008 alloy was solidified with a [001] direction perpendicular to the glass plates, the weak anisotropy in the (001) plane produced dendrites with trunks growing along a [100] direction with [010] side arms. When the seed crystal was oriented in such a way that 2-D growth occurred in the (111) plane, the anisotropy normally vanished and the solid grew as an "oriented seaweed." Finally, when growth occurred again in the (001) plane but at higher speed, a "doublon" morphology was observed. This morphology is similar to a dendrite growing along [100] with [010] side arms, but with a deep liquid channel at the center. Although very carefully conducted, such experiments present two inherent difficulties in their interpretation: first, the influence of the glass plates on the 2-D growth is difficult to assess and second, the contribution of the attachment kinetics of molecules to the solidliquid interface can compete with that related to the solid-liquid interfacial energy $\gamma_{s l}$. The second remark also applies to observations made by Chan et al. ${ }^{[4]}$ in the $\mathrm{NH}_{4} \mathrm{Cl}-\mathrm{H}_{2} \mathrm{O}$ system. In this case, for an increasing supersaturation (or velocity), $\langle 100\rangle$ dendrites were first observed, then $\langle 110\rangle$ trunks with $\langle 100\rangle$ side arms, and finally $\langle 111\rangle$ trunks.

The advantage of metallic alloys is that the contribution of the attachment kinetics to the dendrite growth kinetics and directions is very small for a velocity of the interface in the range of $\mathrm{mm} / \mathrm{s}$ and below. Indeed, in the case of Al-Zn, the same dendrite orientations were identified in specimens directionally solidified (DS) at about $1 \mathrm{~mm} / \mathrm{s}$ and grown in a 
Bridgman furnace at $0.067 \mathrm{~mm} / \mathrm{s}^{[1]}$ Therefore, the DOT in this case was interpreted on the basis of a change of the interfacial solid-liquid energy anisotropy alone. $^{[1,5]}$ Looking at the equilibrium shape of liquid droplets surrounded by the solid, the anisotropy of $\gamma_{s l}$ in $\mathrm{Al}-\mathrm{Cu}$ specimens was estimated to be as low as $1 \mathrm{pct}$ by Napolitano et al. ${ }^{[6,7]}$ Therefore, the strength and anisotropy of $\gamma_{s l}$ can be changed easily in Al-Zn by an increasing amount of zinc, since this hcp element has a much more anisotropic interfacial energy $\gamma_{s l}{ }^{[8]}$ and a very high solubility in fcc aluminum at high temperature (about $83 \mathrm{wt}$ pct). ${ }^{[9]}$

In order to consolidate this interpretation, it is necessary to directly measure the interfacial energy anisotropy in the Al- $\mathrm{Zn}$ system close to the maximum solubility. While trying to achieve this goal, we address in the present article a few questions related to the dendrite or seaweed growth morphologies. The first question is related to the influence of a $\langle h k 0\rangle$ (and more generally $\langle h k l\rangle$ ) dendrite growth direction on the texture evolution in DS specimens. The case of $\langle 100\rangle$ dendrites is well known since the pioneer work of Walton and Chalmers. ${ }^{[10]}$ These authors established a link between the evolution of the density and the gradual $\langle 100\rangle$ texturing of columnar grains as a function of the distance from the mold wall. They showed that grains having a $\langle 100\rangle$ direction misaligned with the thermal gradient have to grow faster, and are thus characterized by a larger undercooling. As they stand slightly behind well-oriented dendrites, their growth is blocked at grain boundaries where the two $\langle 100\rangle$ dendrite trunks are converging, whereas multiple branching occurs at grain boundaries where they diverge. Consequently, such misoriented grains are gradually eliminated as growth proceeds, as shown by in-situ observations in organics. ${ }^{[11]}$ More recently, Gandin et al. ${ }^{[12]}$ have made EBSD measurements on a DS Inconel X750 alloy, which have given more quantitative data on the density and texture evolutions of columnar grains. Such variations could be simulated with a cellular automaton (CA), assuming a random orientation of the nuclei formed at the chill plate surface and $\langle 100\rangle$ dendrite growth directions. The same type of analysis is performed here for various concentrations of zinc, i.e., for various $\langle h k 0\rangle$ dendrite growth directions.

The second question is related to seaweed morphologies: do we observe a similar texturing in DS Al-Zn alloys having a nominal composition close to the start and end of the DOT? This question has absolutely no answer yet as this type of morphology has only been observed under 2-D growth conditions for organic substances. ${ }^{[3]}$ In parallel to three-dimensional (3-D) serial sectioning of such seaweed structures, the answer to such a question can elucidate their growth mechanism.

\section{EXPERIMENTS}

As the alloy preparation, the DS setup and the analysis methods are the same as those reported in Reference 1, they will be described only briefly in the present article. Aluminum-zinc samples of various composition $\left(C_{\mathrm{Zn}}=25,35,50,55\right.$, and $70 \mathrm{wt}$ pct $)$ were prepared from pure aluminum (99.995 pet purity) and zinc ( 99.995 pet purity). After mixing the right proportions of each metal in the liquid state, the alloy was poured in the DS steel mold. Covered with a thin boron nitride layer and surrounded on the lateral side by ceramic wool, the mold was preheated to a temperature $50{ }^{\circ} \mathrm{C}$ above the liquidus before pouring. The whole system was then allowed to rest 10 minutes in a furnace in order to eliminate the convection associated with pouring. After fixing the DS setup to a platform, a water jet was turned on at the bottom of the system until solidification was complete.

After solidification, each DS specimen was cut along a longitudinal midsection, i.e., perpendicular to the chill surface. One halve was dedicated to optical microscopy and EBSD analyze in a longitudinal section, while the other part was cut transversely at various heights from the chill. These longitudinal and transverse sections were mechanically polished on increasingly fine $\mathrm{SiC}$ papers (from 220 to 2400 grades) using water as lubricant, before a final polishing with $6-$ and $1-\mu \mathrm{m}$ diamond spray DP-Mol (Struers GmbH, Willich, Germany) using clothes and ethanol as a lubricant. For EBSD measurements, the surface was electropolished in order to remove the small deformed layer due to mechanical polishing and to obtain a good contrast and indexation of the pseudo-Kikuchi lines (A2 solution $(72 \mathrm{~mL}$ ethanol, $20 \mathrm{~mL}$ 2-buthoxyethanol, and $8 \mathrm{~mL}$ perchloric acid (71 pet concentration)); voltage 10 to $50 \mathrm{~V}$, time 5 to 10 seconds). The EBSD observations were performed on a PHILIPS* XL30FEG SEM microscope with the

*Philips is a trademark of Philips Electronics Instruments Corp., Mahwah, NJ.

following parameters: acceleration voltage 20 to $25 \mathrm{kV}$, spot size $4 \mathrm{~nm}$, working distance 20 to $25 \mathrm{~mm}$, and step size of the displacement stage 10 to $20 \mu \mathrm{m}$. The recording and indexing of the pseudo-Kikuchi lines were made with the software Channel 5 from HKL Technology. The precision of such a system was estimated to be $\pm 2 \mathrm{deg}$ on the orientation measurement (1 deg in relative precision). For each measurement, a small silicon reference with a (001) surface and a horizontal [110] direction was glued on the Al-Zn section for calibration purpose.

\section{GRAIN SELECTION MECHANISM FOR $\langle h k l\rangle$ DENDRITES}

Unlike cellular structures for which the growth direction is essentially that opposite to the heat flow, dendrites grow along preferential crystallographic directions. For metallic alloys solidifying at low growth rate, these directions are dictated by the anisotropy of the solid-liquid interfacial energy $\gamma_{s l}$. Assuming that dendrites are initiated from the most highly curved parts of the equilibrium shape crystal, their directions can be 
obtained from the so-called $\xi$-plot once $\gamma_{s l}(\mathbf{n})$ is known, where $\mathbf{n}$ is the unit normal. ${ }^{[13]}$ As shown by Cahn and Hoffmann, ${ }^{[13]}$ the vector $\xi(\mathbf{n})$ given by $\nabla\left(r \gamma_{s l}(\mathbf{n})\right)$, where $r$ is the radius in spherical coordinates, outlines a surface isomorphous to the equilibrium shape crystal. As the $\xi$ vector formalism retrieves Herring's relations, ${ }^{[14]}$ the most highly curved parts or the equilibrium shape crystal also correspond to minima of the interface stiffness, given by $\left(\gamma_{s l}+\Delta \gamma_{s l}\right)$, where $\Delta$ is the Laplacian.

Let us assume that these preferential dendrite growth directions are given by $\langle h k l\rangle$. In general, there are therefore 48 possible dendrite growth directions, resuming to 24 if $l=0$ (i.e., $\langle k h 0\rangle$ ), to 12 if $l=0$ and $h=k$ (i.e., $\langle 110\rangle$ ), and to 6 if $k=l=0$ (i.e., $\langle 100\rangle$ ). Assuming that the nuclei formed at the bottom surface of the steel mold are randomly oriented, one needs to consider the direction $\langle h k l\rangle$ closest to the thermal gradient in order to see how the grain competition will operate. As done by Gandin et al. for $\langle 100\rangle$ dendrite growth, ${ }^{[12]}$ it is necessary to consider the elementary or unit triangle formed by the directions [100], [110], and [111] (Figure 1). In this triangle, there is a unique [hkl] direction, which appears as a point. We need then to calculate the probability $d p_{h k l}(\theta)$ of finding the thermal gradient $\mathbf{G}=\nabla T$ at an angle $[\theta, \theta+d \theta]$ to the direction $[h k l]$. On the unit surface, the function $p_{h k l}^{\prime}(\theta)=$ $d p_{h k l}(\theta) / d \theta$ is given by the arc length of the cone of axis $[h k l]$ and aperture $\theta$ (called hereafter "cone $\theta_{h k l}$ "), contained within the unit triangle and divided by the surface of the unit triangle, i.e., $\pi / 12$. This probability, which is calculated in more detail in the Appendix, will be compared with the EBSD statistics measured for each specimen near the chill surface.

Please note that $p_{h k l}^{\prime}(\theta)$ varies between 0 and a maximum $\theta_{\max } \leq \arccos (1 / \sqrt{3})$ (the equal signs holds when $[h k l=[100]$ or [111]). Close to $\theta=0$, the distribution $p_{h k l}^{\prime}(\theta)$ varies as $(\sin \theta)$ regardless of the

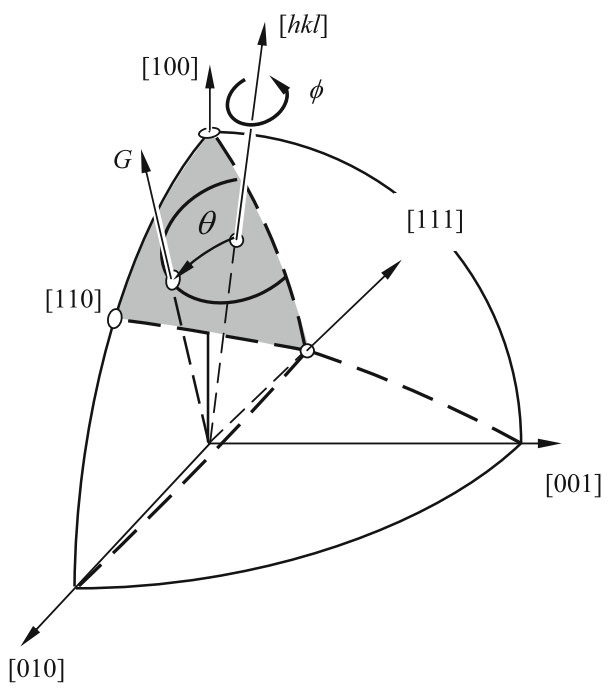

Fig. 1-One quadrant of the unit sphere and unit [100]-[110][111] triangle (gray area). The probability $p_{h k l}^{\prime}(\theta)$ of finding the gradient $G$ at an angle $\theta$ from an $[h k l]$ direction is given within this triangle by the arc length intersected on the sphere by the cone $\theta_{h k l}$, i.e., the cone of axis $[h k l]$ and aperture $\theta$, normalized by the surface $\pi / 12$ of the unit triangle. direction $[h k l]$ considered in the unit triangle. However, for a direction near the center of the unit triangle, it varies as $(24 \sin \theta)$ as the angle $\phi$ varies between 0 and $2 \pi$. For a $[h k 0]$ growth direction, it varies as $(12 \sin \theta)$ if $h \neq k$, as $(6 \sin \theta)$ if $h=k=1$ and as $(3 \sin \theta)$ if $h=1$ and $k=0$. One retrieves the multiplicity factor of the directions $(48: 24: 12: 6$ for $\langle h k l\rangle:\langle h k 0\rangle:\langle 110\rangle:\langle 100\rangle)$. However, for a general direction $\langle h k l\rangle$, the value $\theta_{\max }$ will be reduced, i.e., the random distribution of nuclei close to the chill $p_{h k l}^{\prime}(\theta)$ will be narrower than the corresponding $p_{100}^{\prime}(\theta)$. In summary, the distribution $p_{h k l}^{\prime}(\theta)$ varies between 0 and $\theta_{\max }$ given by the maximum angle between $[h k l]$ and the three corners of the unit triangle. It reaches a maximum probability for an angle $\theta_{\text {opt }}$ corresponding to the maximum arc length intersected by the cone $\theta_{h k l}$ with the unit sphere and contained within the unit triangle.

Under steady-state conditions, $\langle h k l\rangle$ dendrite trunks, which are misoriented by an angle $\theta$ with respect to the thermal gradient, have a velocity $v_{\theta}=v_{T} / \cos \theta$, where $v_{T}$ is the velocity of the (liquidus) isotherm. Therefore, the tip undercooling $\Delta T\left(v_{\theta}\right)$ is an increasing function of $\theta$.

According to the minimum undercooling mechanism outlined by Walton and Chalmers, ${ }^{[10]}$ one expects the grains having the largest misorientation, i.e., located near $\theta_{\max }$ in the distribution $p_{h k l}^{\prime}(\theta)$, to be eliminated first. Therefore, as columnar growth proceeds, the distribution $p_{h k l}^{\prime}(\theta)$ will become narrower around an optimum $\theta_{\text {opt }}$ getting closer to 0 as the distance to the chill increases. These evolutions are shown in the next section for both the dendritic and seaweed specimens.

\section{RESULTS AND DISCUSSION}

From the analysis made in Reference 1, the growth directions listed in Table I were obtained for DS Al-Zn specimens of various concentrations. These orientations were confirmed in the present analysis. It should be emphasized that the "growth direction" is that of the dendrite trunks for "regular" dendritic specimens, while for seaweeds, it corresponds to the texture of the specimen after some selection among the grains has operated.

In order to analyze the grain competition that occurs in these specimens as solidification progresses, we present the results in two parts: first, the texture evolution and second, the evolution of the grain density as measured in transverse cross sections.

Table I. Growth Directions Observed in DS Specimens as a Function of the Zinc Composition

\begin{tabular}{lc}
\hline $\mathrm{C}_{\text {zn }}$ (Wt Pct) & Growth Direction \\
\hline 25 & $\langle 100\rangle$ \\
35 & $\langle 210\rangle$ seaweed \\
50 & $\langle 320\rangle$ \\
55 & $\langle 110\rangle$ seaweed \\
70 & $\langle 110\rangle$ \\
\hline
\end{tabular}




\section{A. Texture Evolution}

For each composition, the EBSD results are presented in Figures 2 through 4, 6, and 7 according to an identical template:

- First, a reconstructured false color EBSD map of a longitudinal section from the chill zone to a height of about 6 to $10 \mathrm{~mm}$ is presented in Figure 2(a). The color code corresponds to the angle $\theta$ between the vertical thermal gradient $G$ and the closest $\langle h k 0\rangle$ growth direction shown in Table I. The grain boundaries are represented providing the misorientation between two neighbor grains is larger than $5 \mathrm{deg}$.

- Based on the EBSD measurements performed on transverse sections, a statistic of the pixel orientation $p_{h k 0}^{\prime}(\theta)$ is presented in Figures 2(b) through 4(b), 6(b), and 7(b) for various heights $z$ in the specimen. Again, the angle $\theta$ measures the misorientation between the thermal gradient $G$ and the closest [hk0] direction. The statistics measured close to the chill plate (identified as $0 \mathrm{~mm}$ in the figures) is compared with the statistics computed from a random orientation distribution (the Appendix provides details). Please note that each curve $p_{h k 0}^{\prime}(\theta)$ is normalized to unity, i.e., the integral of this distribution over the angle $\theta$ is equal to 1 .

Finally, the corresponding $\langle h k 0\rangle$ pole figures measured on a few transverse sections at different heights are shown in Figures 2(c) through 4(c), 6(c), and 7(c). The gradient being placed at the center of these pole figures, the color code is identical to that used in the longitudinal section, i.e., color corresponding to the angle $\theta$ between $G$ and the closest [hk0] direction. In these figures, an "ideal" stereographic projection of a family of grains having one [hk0] direction perfectly aligned along the gradient, i.e., at the center of the pole figure, but with a random rotation around this axis might also be shown for comparison.

Starting with the lowest zinc-content specimen $\left(C_{\mathrm{Zn}}=25 \mathrm{wt}\right.$ pct, Figure 2$)$ for which standard $\langle 100\rangle$

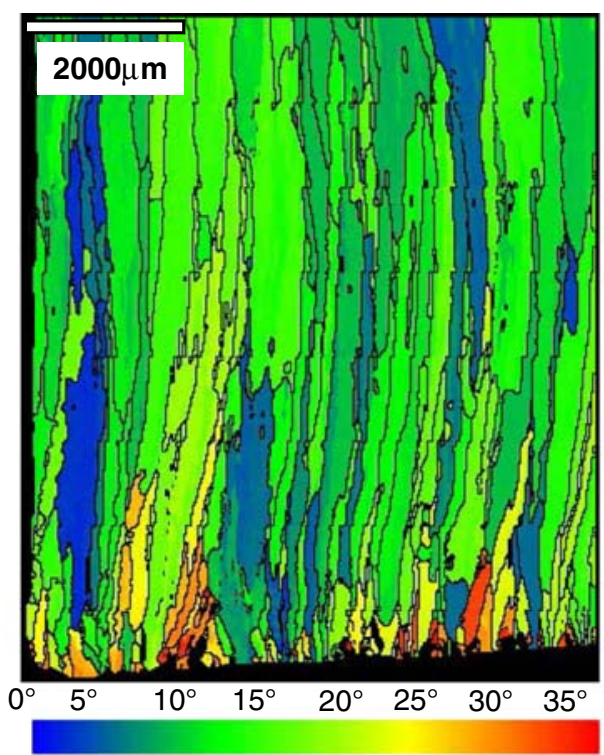

(a)

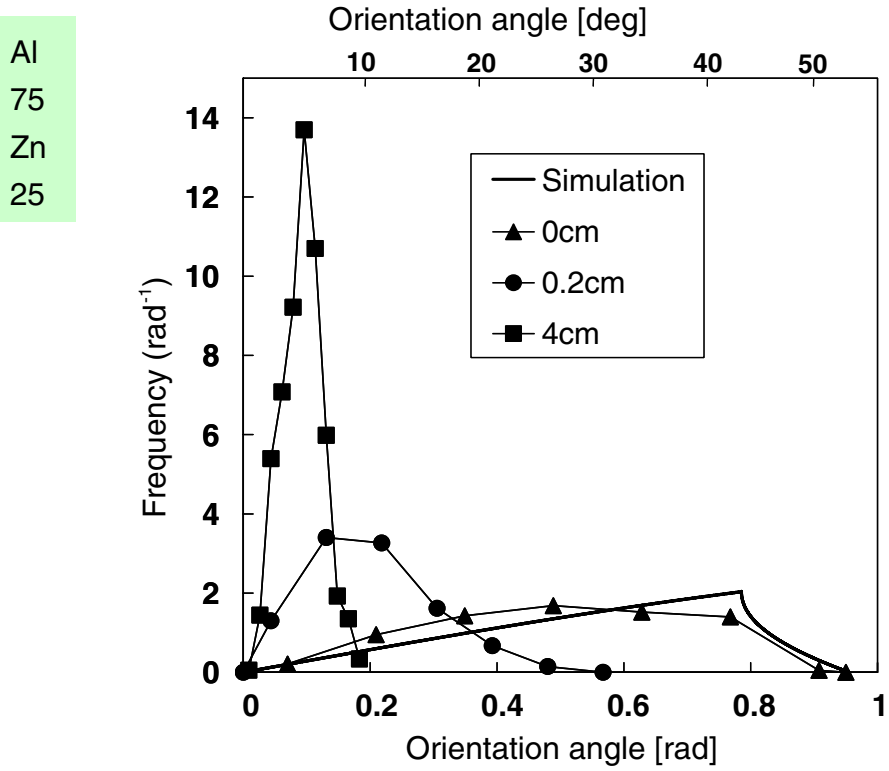

(b)

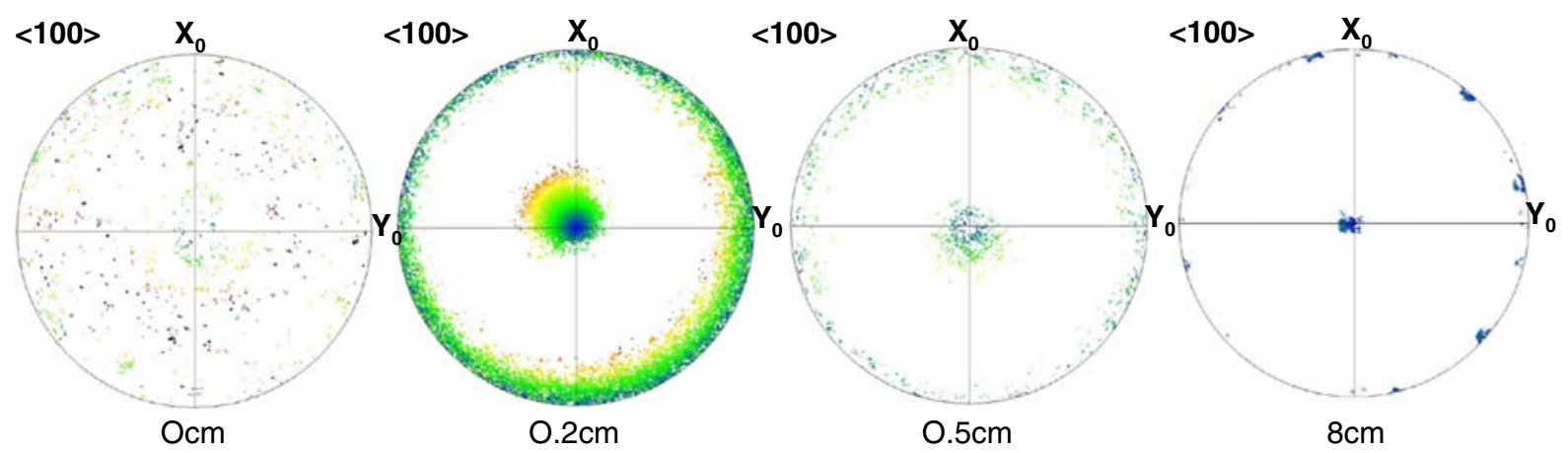

(c)

Fig. 2-Al-25 wt pet $\mathrm{Zn}(a)$ EBSD false color map of the grain structure in the longitudinal section, (b) statistics of the orientation distribution $p_{100}^{\prime}(\theta)$, and $(c)$ pole figures at various heights. 

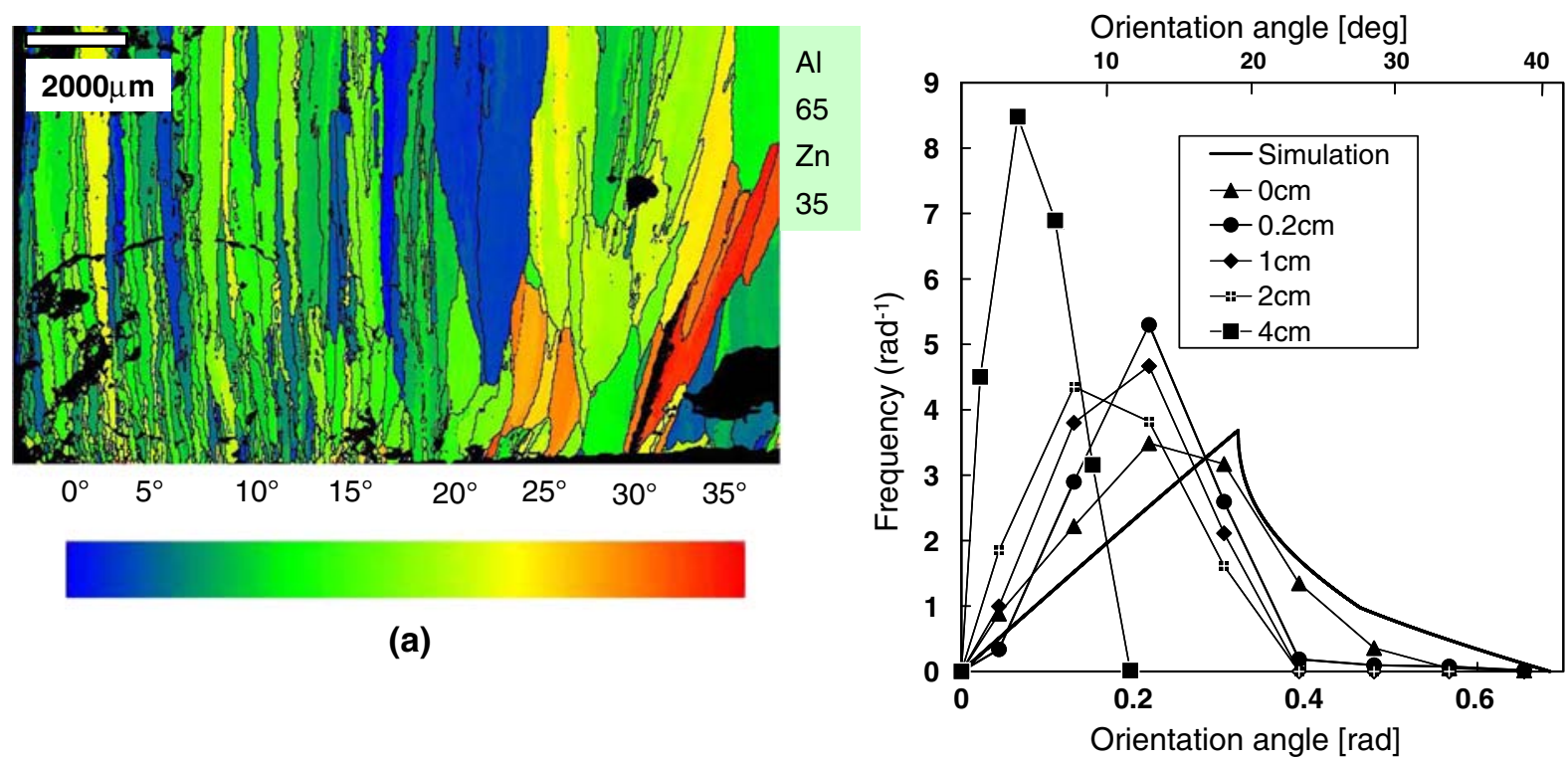

(b)
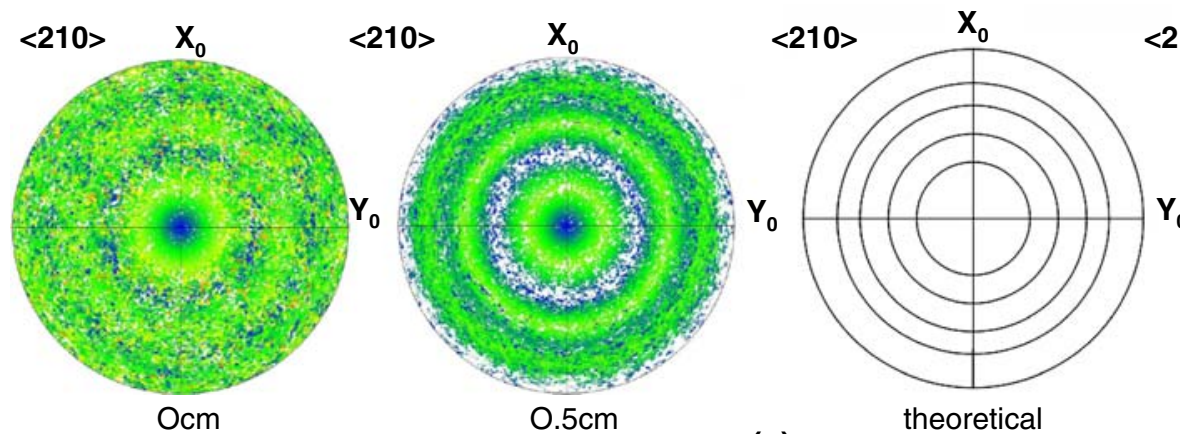

(c)

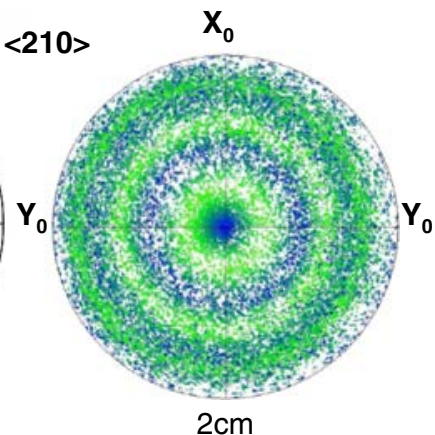

Fig. 3-Al-35 wt pet Zn (a) EBSD false color map of the grain structure in the longitudinal section, (b) statistics of the orientation distribution $p_{210}^{\prime}(\theta)$, and $(c)\langle 210\rangle$ pole figures at various heights.

dendrites are expected, the distribution of the nuclei close to the chill surface appears to be nearly random. This can be seen from the fairly uniform scattering of the points in the $\langle 100\rangle$ pole figure taken at height zero (shown in Figure 2(c)). Looking at the corresponding statistics in Figure 2(b) (curve with filled triangles), the measured distribution $p_{100}^{\prime}(\theta)$ at $(z=0)$ indeed follows quite well the theoretical distribution (curve without symbol labeled "simulation"). It is already slightly moved toward lower $\theta$ values as it is difficult to make an EBSD measure on an unpolished bottom surface of the casting. So, a small layer was removed during the polishing operation. In the longitudinal false color micrograph shown in Figure 2(a), the grains located near the chill appear with different colors, from blue $(\langle 100\rangle$ parallel to $G)$ to red (misorientation of $35 \mathrm{deg}$ ). Although the maximum misorientation for $\langle 100\rangle$ growth direction is $\arccos (1 / \sqrt{3})=54.7 \mathrm{deg}$, this limited scale was chosen so as to see some color variations in the remaining of the specimen. Indeed, as can be seen in this longitudinal section, most of the grains that survive after a few $\mathrm{mm}$ of growth are green, with a few blue ones. This is confirmed in the pole figures in Figure 2(c), which already show a strong $\langle 100\rangle$ texturing at $2 \mathrm{~mm}$ from the chill. Please note that the EBSD points at $z=2 \mathrm{~mm}$ are not exactly centered in the projection and this is also reflected by the two other $\langle 100\rangle$ directions at the periphery of the projection (the annulus of points are slightly tilted toward the north-west quadrant). This is most probably due to slightly inhomogeneous cooling conditions at the chill surface. Indeed, at $5 \mathrm{~mm}$ and $8 \mathrm{~cm}$, further selection has occurred and the points are now perfectly centered. Please note also that fewer grains are available in the transverse sections, as will be described in more detail in Section B.

One might be surprised that the longitudinal section in Figure 2(a) remains essentially green instead of blue. However, as already pointed out by Gandin et al., ${ }^{[12]}$ this is expected. As the orientation distribution $p_{100}^{\prime}(\theta)$ varies as $(\sin \theta)$ close to $\theta=0$, the probability of having a $\langle 100\rangle$ direction exactly aligned along $G$ is zero. This is confirmed in the statistics shown in Figure 2(b). As one goes away from the chill, the distribution gets narrower but the maximum is still 5 deg off the thermal gradient at a height $z=4 \mathrm{~cm}$ (only three curves $p_{100}^{\prime}(\theta)$ at $z=0$, 0.2 , and $4 \mathrm{~cm}$ are shown for clarity). 


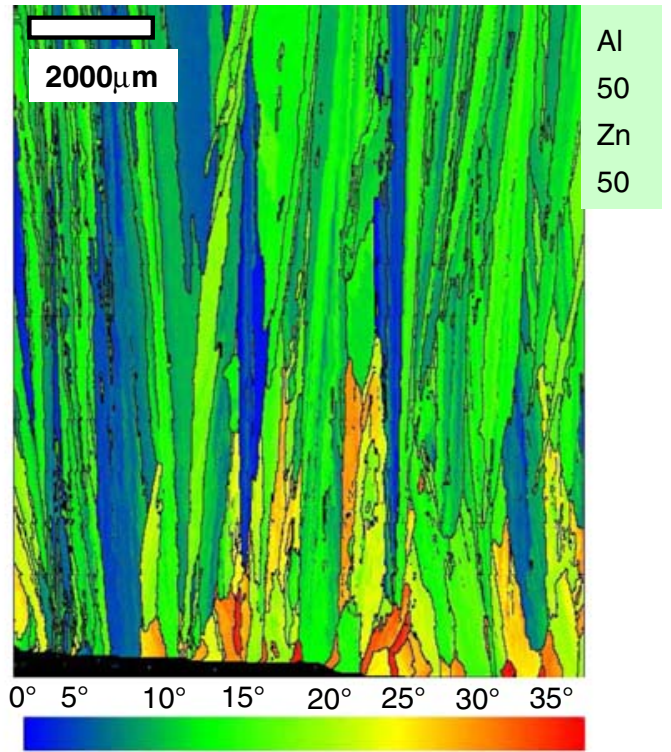

(a)

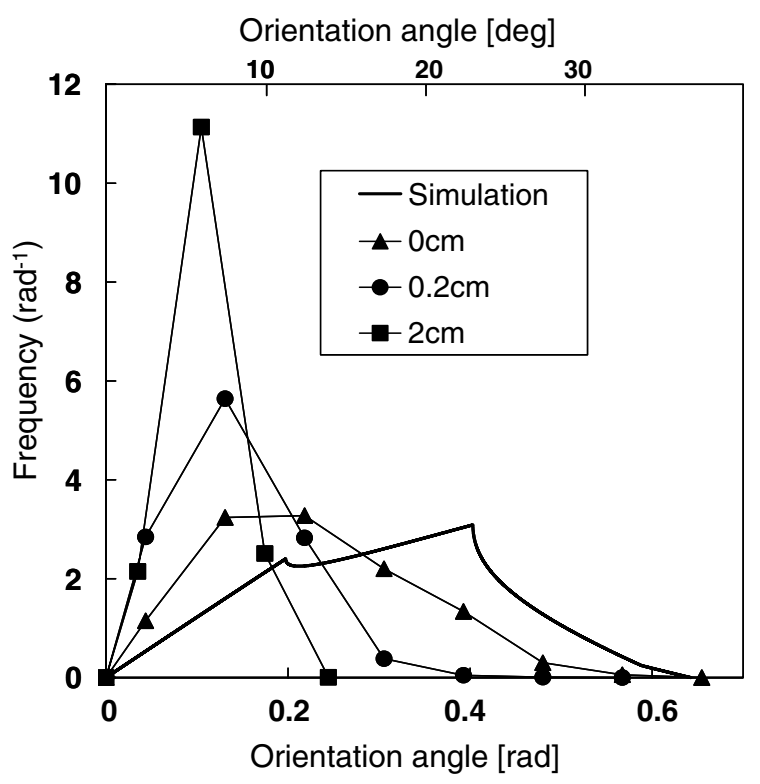

(b)
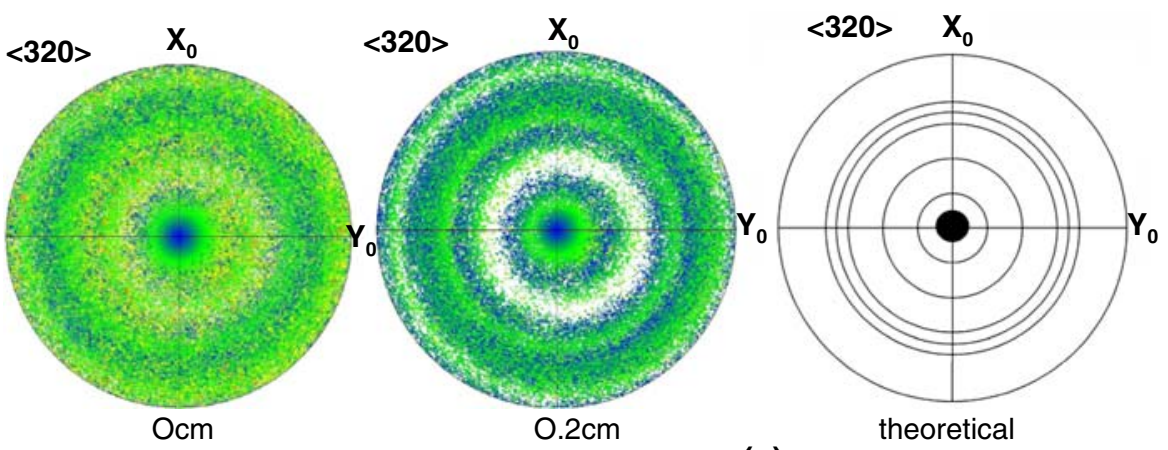

(c)

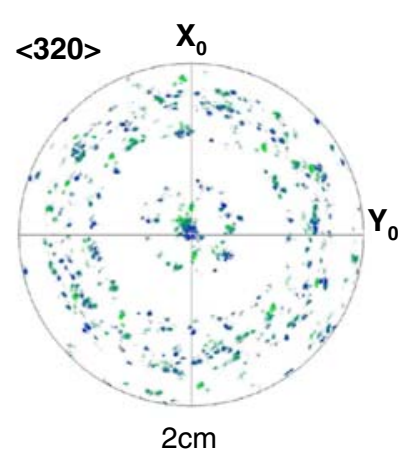

Fig. 4-Al-50 wt pct $\mathrm{Zn}(a)$ EBSD false color map of the grain structure in the longitudinal section, $(b)$ statistics of the orientation distribution $p_{320}^{\prime}(\theta)$, and $(c)\langle 320\rangle$ pole figures at various heights.

Not surprisingly, the grain competition based on the minimum undercooling criterion (or the smallest velocity, or the best alignment with the thermal gradient) does seem to operate in this Al-25 wt pct Zn specimen. Let us now consider the seaweed specimen Al-35 wt pct $\mathrm{Zn}$ for which a $\langle 210\rangle$ texturing direction was already reported in Reference 1. As shown in Figure 3(a), texturing is indeed occurring despite the fact that one has seaweeds and not dendrites in this specimen. The color code for this longitudinal micrograph is again according to the angle between the thermal gradient $G$ (vertical) and the closest $\langle 210\rangle$ direction. The maximum misorientation, which corresponds to $G$ parallel to $\langle 111\rangle$ ( $39 \mathrm{deg}$ ), is now close to the maximum of the color scale. Close to the surface of the chill, the grain statistics in a transverse section shown in Figure 3(b) follow fairly well the calculated distribution. However, the angle corresponding to the maximum probability is only $18 \mathrm{deg}$ in this case. This explains why the corresponding pole figure $z=0$ in Figure 3(c) shows a random coverage of EBSD points but mainly colored in green. The blue points near the center correspond to grains for which $G$ is parallel to a $\langle 210\rangle$ direction, the other $\langle 210\rangle$

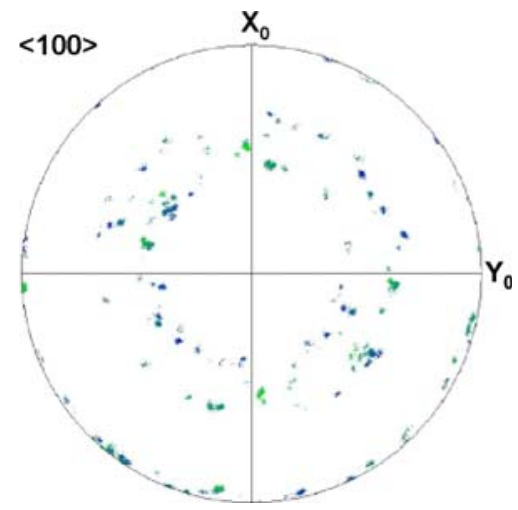

Fig. 5- $\langle 100\rangle$ pole figure made on the transverse section at $2 \mathrm{~cm}$ from the bottom of the Al-50 wt pet Zn DS specimen, with the thermal gradient $G$ at the center.

directions of such grains appearing also blue on rings located at 36.9, 53.1, 66.4, 78.5, and $90 \mathrm{deg}$ from the center. The circles corresponding to these values are reported in the ideal stereographic projection shown in Figure 3(c). 


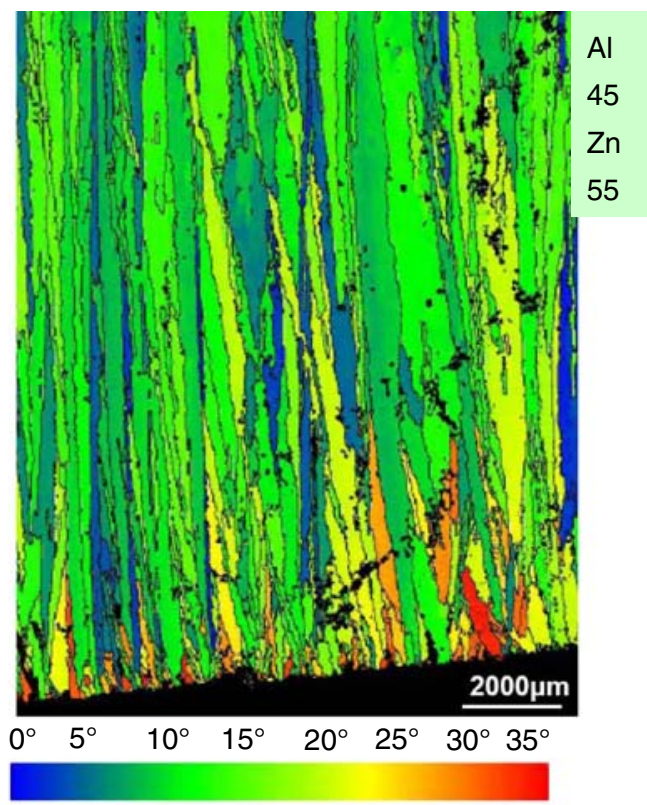

(a)

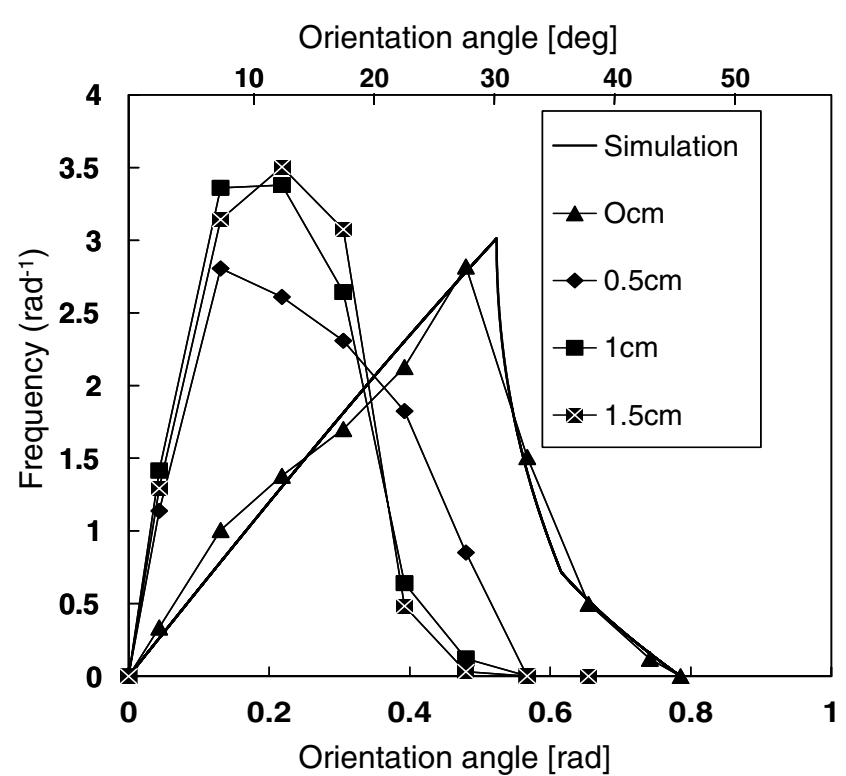

(b)

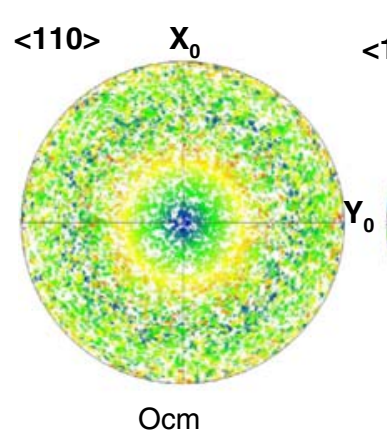

Ocm

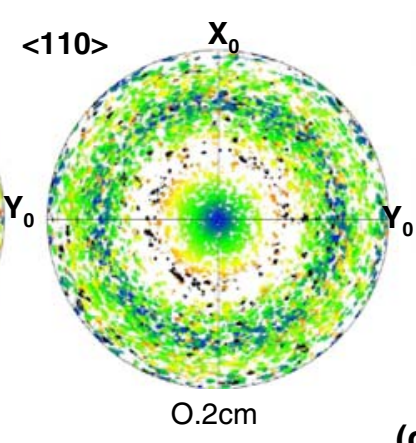

(c)

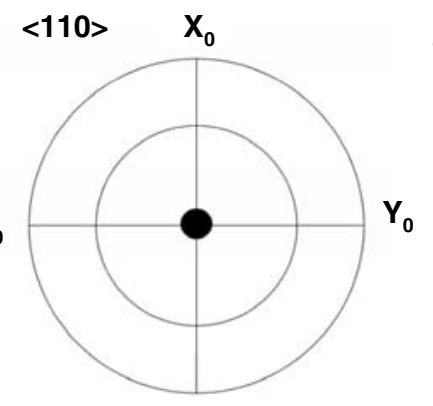

theoretical

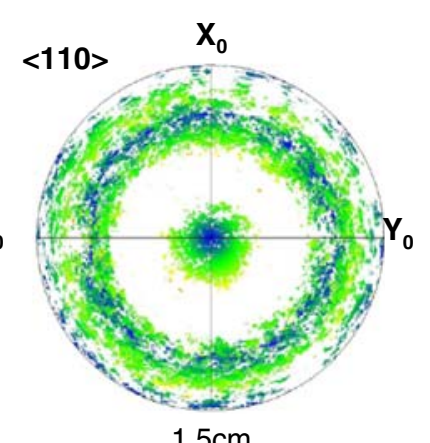

$1.5 \mathrm{~cm}$

Fig. 6-Al-55 wt pet Zn (a) EBSD false color map of the grain structure in the longitudinal section, (b) statistics of the orientation distribution $p_{110}^{\prime}(\theta)$, and $(c)\langle 110\rangle$ pole figures at various heights.

There are also a few misoriented grains in the longitudinal section of Figure 3(a) and appearing in red. They do seem to grow over longer distances compared to the dendritic $\langle 100\rangle$ specimen (Figure 2(a)). Indeed, as indicated by the statistics $p_{210}^{\prime}(\theta)$ in Figure 3(b), the overall grain selection in the seaweed specimen is not as effective: the curves for $z=0.2,1$, and $2 \mathrm{~cm}$ are slightly shifted toward lower $\theta$ values, but not to the same extent as in the dendritic specimen. This will be seen also in the grain density evolution (Section IV-B). Only at $4 \mathrm{~cm}$ does the curve exhibit a statistics ranging from 0 to $12 \mathrm{deg}$, with a maximum around $5 \mathrm{deg}$. The same selection is observed in the pole figures in Figure 3(c), but due to the multiplicity of the $\langle 210\rangle$ directions the regions that are free of EBSD points are greatly reduced. Nevertheless, the remaining points are more and more blue and located within the possible angles determined from a minimum angle between $G$ and the closest $\langle 210\rangle$ direction.

The multiplicity of the $\langle 210\rangle$ directions is partially responsible for the less effective texture evolution. Indeed, the maximum misorientation of a grain with respect to the thermal gradient is only 39 deg (compared to 54.7 for $\langle 100\rangle)$ and the maximum of $p_{120}^{\prime}(\theta)$ occurs near $18 \mathrm{deg}$ (compared to $45 \mathrm{deg}$ for $p_{100}^{\prime}(\theta)$ ). However, this might be not the only reason, as one shall see when comparing a $\langle 110\rangle$ seaweed (Figure 6) and a $\langle 110\rangle$ dendritic specimen (Figure 7). This reduced selection is probably also linked with the morphology of the microstructure itself.

Looking now at the $50 \mathrm{wt}$ pct specimen (Figure 4) for which $\langle 320\rangle$ dendrites have been observed, ${ }^{[1,15]}$ similar features can be seen. Interestingly, the distribution of a random population of grains $p_{320}^{\prime}(\theta)$ shows a local maximum near $11 \mathrm{deg}$ and an absolute maximum around $23 \mathrm{deg}$, the maximum misorientation being $36.8 \mathrm{deg}$. The measured curve shown in Figure 4(b) indicates that some grain selection has already occurred at the section indicated as $z=0$. This is again due to the small polishing required at the bottom surface of the casting, which for some specimens is more important than for others. The grain selection seems to operate well in this specimen despite the multiplicity of the $\langle 320\rangle$ directions and the fairly narrow $p_{320}^{\prime}(\theta)$ initial distribution. At $2 \mathrm{~cm}$ 


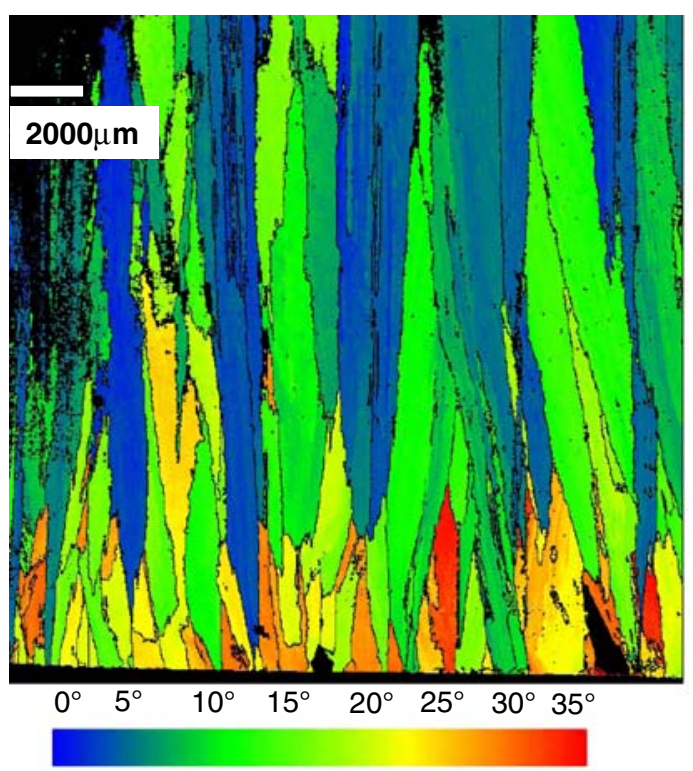

(a)

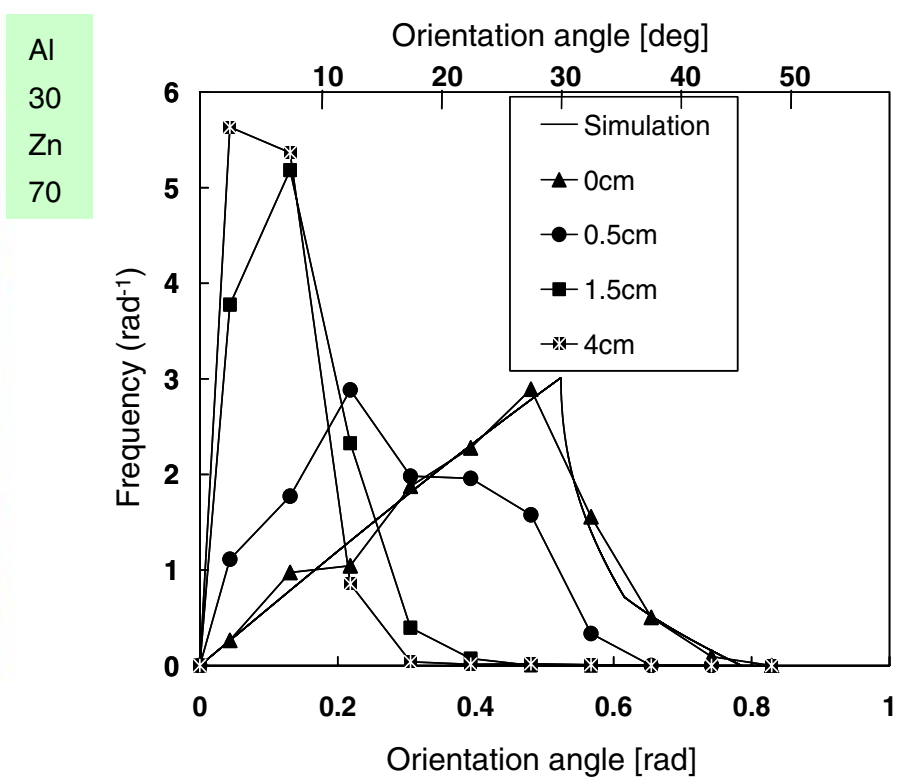

(b)
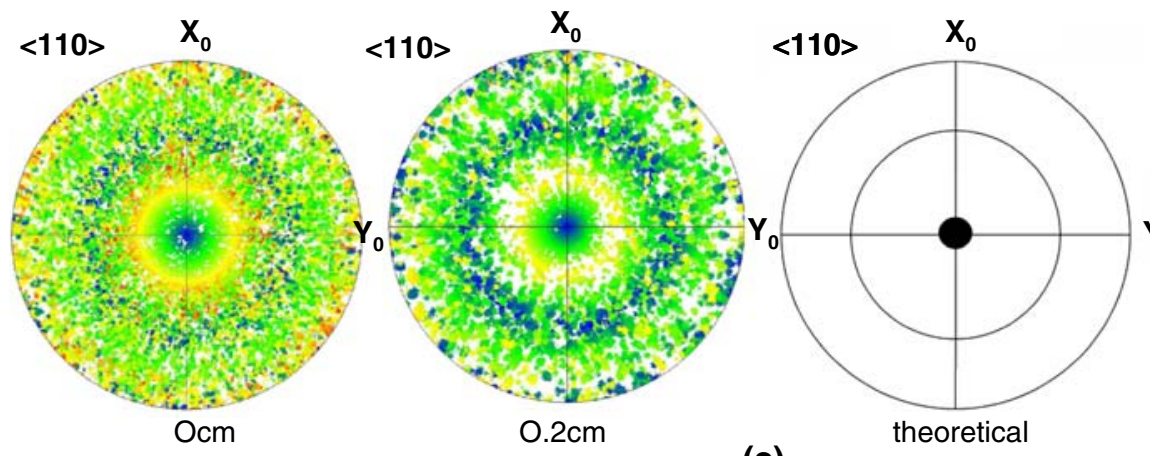

(c)

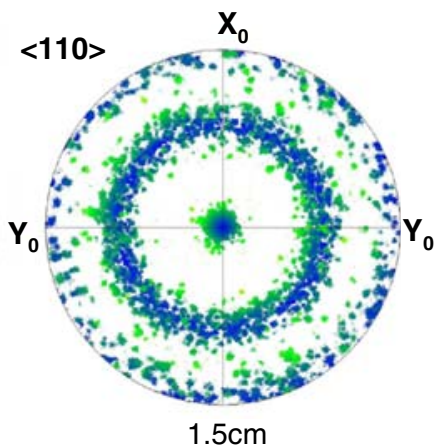

Fig. 7-Al-70 wt pet Zn (a) EBSD false color map of the grain structure in the longitudinal section, (b) statistics of the orientation distribution $p_{110}^{\prime}(\theta)$, and $(c)\langle 110\rangle$ pole figures at various heights.

from the chill, the distribution is centered around $5 \mathrm{deg}$, while this only occurred at $4 \mathrm{~cm}$ for the seaweed $\langle 210\rangle$ specimen (Figure 3 ). This selection is clearly visible in the pole figures of Figure 4(c), especially since the other $\langle 320\rangle$ directions are not as uniformly scattered over $\theta$ as compared with the $\langle 210\rangle$ specimen (Figure 3). Indeed, the possible angles between $\langle 320\rangle$ directions are 22.6, 46.2, 62.5, 67.4, and $72.1 \mathrm{deg}$; the last three angles appear as three rings fairly close to each other in the ideal stereographic projection, thus making the grain selection more apparent to the viewer.

The texturing of this specimen is fairly effective probably for two reasons: first, the microstructure is made of dendrites and not seaweeds,${ }^{[1,15]}$ and second, the [320] orientation is getting closer to one corner of the unit triangle (it is only 11.3 deg off the [110] direction). As we shall see for the $\langle 110\rangle$ dendritic specimen (Figure 7), grain selection is as effective as for the $\langle 100\rangle$ specimen (Figure 2). One might even ask whether the $\langle 320\rangle$ dendrite growth direction cannot be confused with $\langle 110\rangle$. Figure 5 answers this question in an unambiguous way. In this figure, the $\langle 100\rangle$ pole figure of this
Al-50 wt pet $\mathrm{Zn}$ specimen is displayed for the transverse section made at $2 \mathrm{~cm}$ from the chill. It is clear that texturing has occurred along an $\langle h k 0\rangle$ direction, since an annulus of $\langle 100\rangle$ EBSD points is located at the periphery of the stereographic projection, i.e., $\theta=90 \mathrm{deg}$. On the other hand, the indices $h$ and $k$ cannot be equal since one clearly distinguishes two rings of points in this pole figure. This eliminates de facto $\langle 110\rangle$ texturing. These rings are slightly larger than those corresponding to $\arccos (3 / \sqrt{13})$ and $\arccos (2 / \sqrt{13})$, i.e., the possible angles between $\langle 100\rangle$ and $\langle 320\rangle$, because again the maximum probability $p_{320}^{\prime}(\theta)$ of finding the gradient $G$ (at the center of the projection) aligned with $\langle 320\rangle$ does not occur for $\theta=0$ but for $\theta \approx 6 \operatorname{deg}$ (Figure 4(c)).

Increasing the zinc concentration to $55 \mathrm{wt}$ pct produces again seaweeds as shown in Reference 1, but textured along a $\langle 110\rangle$ direction. The evolution of the texture in such a specimen is shown in Figure 6. Close to the chill, the distribution of nuclei follows a random distribution very precisely (Figure 6(b)). There is again a grain selection as the distance to the chill increases in a 
direction, which is now $\langle 110\rangle$. However, the scattering of the $\langle 110\rangle$ EBSD points shown in the corresponding pole figure (Figure 6(b)) at $1.5 \mathrm{~cm}$ is still quite large. The distribution $p_{110}^{\prime}(\theta)$ at that height is centered around $12 \mathrm{deg}$ and has a full-width at half-maximum (FWHM) of $16 \mathrm{deg}$ (Figures 6(b) and (c)). This situation can be compared with that encountered for the Al-70 wt pct Zn specimen (Figure 7). At the same height, this now dendritic specimen has developed also a $\langle 110\rangle$ texture, but the maximum of $p_{110}^{\prime}(\theta)$ has its maximum around $6 \mathrm{deg}$ and a FWHM of $9.6 \mathrm{deg}$. This clearly shows that texturing is not only a question of the multiplicity of the $\langle h k l\rangle$ growth directions, but also of the growth morphology: the texturing of seaweeds does not occur as rapidly as that of dendritic specimens for the same average preferential growth direction. This finding is confirmed by the evolution of the grain density as a function of the distance to the surface of nucleation.

\section{B. Grain Density Evolution}

The evolution of the grain structure for the five Al-Zn DS specimens investigated in this work can be visualized in Figure 8, where reconstructured false color maps obtained from the EBSD measurements in transverse sections are shown. The grains are again colored according to the angle $\theta$ between the thermal gradient $G$ (perpendicular to these maps) and the closest $\langle h k 0\rangle$ direction listed in Table I. At the surface of the chill (Figure 8(a)), the grains have various colors, ranging from blue to red according to their misorientation with respect to $G$. Their density is fairly high, typically 20 to 60 grains per $\mathrm{mm}^{2}$. Unfortunately, these densities are not totally representative of the real nucleation conditions at the surface of the chill, as some polishing had to be made before the EBSD measurement. Nevertheless, these conditions might differ from one sample to another as seen for the specimen with $C_{\mathrm{Zn}}=50 \mathrm{wt}$ pct. The substantially larger grain density observed in this case (65 grains per $\mathrm{mm}^{2}$ ) is nearly double compared to the other specimens and is definitely beyond the uncertainty associated with the removal of a thin layer of the DS specimen by polishing. Nucleation conditions are influenced therefore by other factors, such as the thickness of the boron nitride coating applied to the chill surface, the local cooling conditions, etc. What is more interesting to look at is the evolution of the grain density in these specimens as growth proceeds.

At $1 \mathrm{~cm}$ from the chill surface (Figure 8(b)), the grain density has decreased substantially due to grain competition. Please note the change of scale (from $500 \mu \mathrm{m}$ in Figure 8(a) to $2000 \mu \mathrm{m}$ in Figure 8(b)). The grains are now essentially blue and green, as already discussed in the previous section. A few yellow grains are still present in the Al-55 wt pet $\mathrm{Zn}$ specimen exhibiting $\langle 110\rangle$ seaweeds, in agreement with the fairly slow texturing of this specimen. This is slightly less obvious in the other seaweed Al-35 wt pet Zn specimen. However, this last specimen clearly exhibits a much finer grain structure at $z=1 \mathrm{~cm}$ and the $2000 \mu \mathrm{m}$ scale had to be adjusted compared with the other specimens. This finer grain size is also representative of the rather slow texturing of this specimen.

In order to quantify the grain competition associated with the texture evolution, the evolution of the grain density, $n_{S}(z)$, was measured by a surface count in transverse sections. The result is shown in Figure 9.

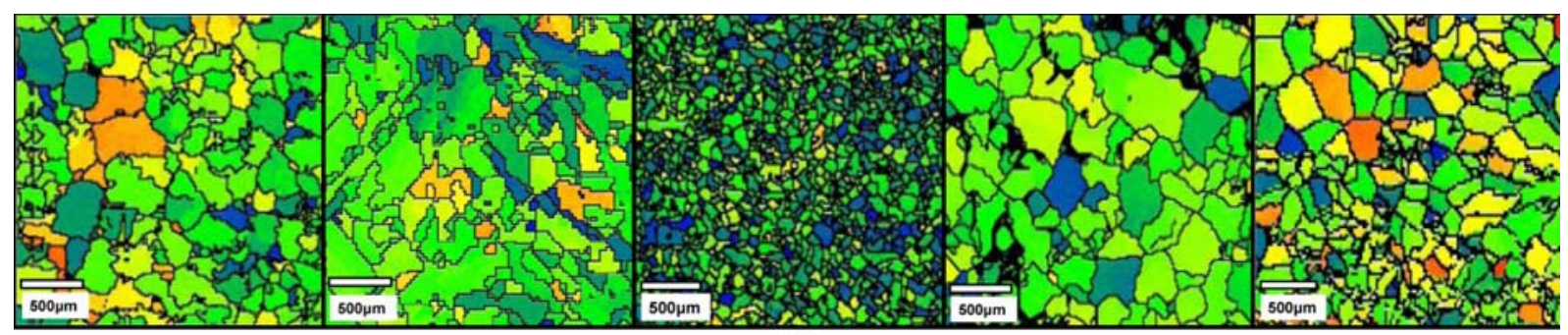

(a)

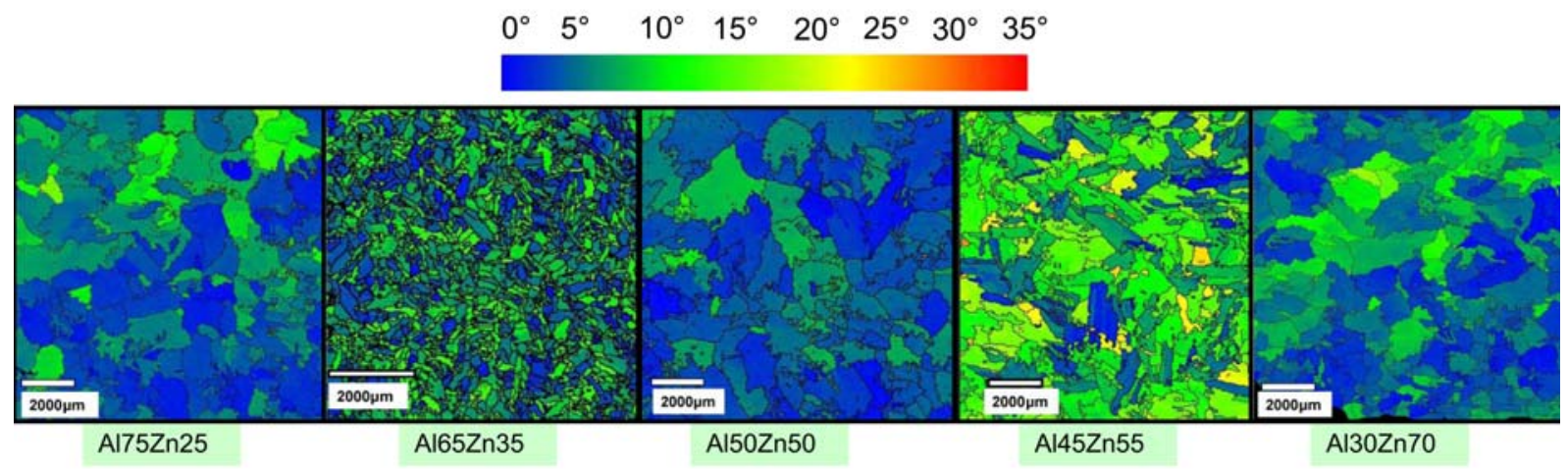

(b)

Fig. 8-Reconstructured false color EBSD map of transverse sections for the six Al-Zn DS specimens $(a)$ near the chill surface and $(b)$ after $1 \mathrm{~cm}$ of growth. 


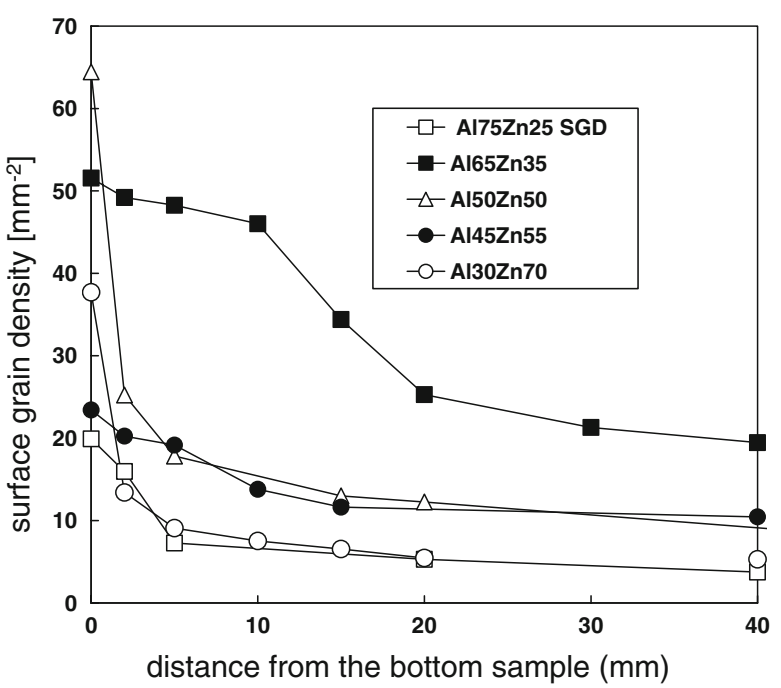

Fig. 9-Surface grain density $n_{S}$ as a function of the distance $z$ to the surface of the chill for the five Al- $\mathrm{Zn}$ compositions.

Please note that the surface used for this analysis $\left(100 \mathrm{~mm}^{2}\right)$ is much larger than the representative areas shown in Figure 8. As already observed by Gandin et al. for $\langle 100\rangle \mathrm{Ni}$ dendrites, ${ }^{[12]}$ the evolution of $n_{S}(z)$ for the dendritic $\mathrm{Al}-\mathrm{Zn}$ specimens is very fast (i.e., for $C_{\mathrm{Zn}}=$ 25,50 , and $70 \mathrm{wt}$ pct, curves with open squares, triangles, and circles in Figure 9, respectively). Then, the density remains fairly constant as the misorientation between grains becomes increasingly small. For example, at $5 \mathrm{~mm}$ from the chill, the corresponding distributions $p_{h k 0}^{\prime}(\theta)$ measured for these three specimens have their maximum at around $10 \mathrm{deg}$ or below, while the largest misorientation is around $20 \mathrm{deg}$ (Figures 2, 4, and 7). For a grain misoriented by $10 \mathrm{deg}$ with respect to its neighbor, the difference in dendrite velocity, given by $\left|v_{T}\left(\cos \theta_{1}\right)^{-1}-v_{T}\left(\cos \theta_{2}\right)^{-1}\right|$ with $\left|\theta_{1}-\theta_{2}\right|=10 \quad \mathrm{deg}$, does not exceed 5 pct. The undercooling of the dendrite tips being roughly a square root function of the dendrite tip velocity, ${ }^{[2]}$ the undercooling difference is reduced to 2.5 pct. For an average undercooling of about $5 \mathrm{~K}$, this represents therefore a $z$-difference of only $40 \mu \mathrm{m}$ in a thermal gradient of $3 \mathrm{~K} / \mathrm{mm} .{ }^{[1]}$ This value is comparable with the secondary dendrite arm spacing, ${ }^{[2]}$ thus explaining why grain competition induced by dendrite tips and arms interactions is greatly reduced below such misorientations.

Assuming an exponential decay of the surface density, i.e., $n_{s}(z)=n_{s \infty}+\left(n_{s 0}-n_{s \infty}\right) \exp (-\mathrm{z} / \delta)$ and making a least-squares fit on the curves shown in Figure 9, the characteristic "competition length" $\delta$ found for the $\langle 100\rangle,\langle 320\rangle$, and $\langle 110\rangle$ dendritic specimens is to 2.2, 2.0, and $2.0 \mathrm{~mm}$, respectively (Table II). Looking at the two seaweed specimens (filled squares and circles for $C_{\mathrm{Zn}}=$ 35 and 55 wt pet in Figure 9), the decay of $n_{S}(z)$ is much slower. This is particularly visible for the specimen exhibiting $\langle 210\rangle$ seaweeds as it has a fairly large initial density. Assuming again an exponential decay, one has $\delta=3.7$ and $4.3 \mathrm{~mm}$ for the $35 \mathrm{wt}$ pct (i.e., $\langle 210\rangle$ ) and $55 \mathrm{wt}$ pct (i.e., $\langle 110\rangle$ ) seaweed specimen, respectively
Table II. Critical Distance of Grain Competition $\delta$ and Mean Aspect Ratio of the Grains $\bar{E}$ Observed in the Transverse Sections of DS Al-Zn Specimens of Various Compositions

\begin{tabular}{lcc}
\hline Composition & $\delta(\mathrm{mm})$ & $\bar{E}(-)$ \\
\hline Al-25 wt pet Zn & 2.2 & 1.1 \\
Al-35 wt pct Zn & 3.7 & 1.9 \\
Al-50 wt pet Zn & 2.0 & 1.4 \\
Al-55 wt pet Zn & 4.3 & 1.9 \\
Al-70 wt pet Zn & 2.0 & 1.2 \\
\hline
\end{tabular}

(Table II). This slower decay of the grain density in seaweed specimens is accompanied by (or due to) a change of the grain morphology in transverse section, as analyzed in more details in Section IV-C.

\section{Seaweed Texture and Morphology}

As is clearly visible in Figure 8(b), the shape of the grains in the transverse section taken at $1 \mathrm{~cm}$ is fairly isotropic for dendritic specimens, i.e., for $C_{\mathrm{Zn}}=25,50$, and $70 \mathrm{wt}$ pct. In order to quantify the aspect ratio (or elongation factor) $E$ of columnar grains in transverse cross sections, an ellipse was fitted on each of them. From this shape, the maximum and minimum ferrets of each grain were deduced, their ratio giving the aspect ratio $E(E \geq 1)$. This analysis was made using the package of the HKL EBSD software. The statistics of the aspect ratio $E(z)$ measured on the transverse sections of the dendritic $\langle 320\rangle$ specimen is shown in Figure 10(b). The number $E_{j}$ indicated for each class $(j)$ has been used to rank the measured values $E(z)$ in each section according to $E_{j-1}<E(z) \leq E_{j}$ (for the first class, one has obviously $\left.1 \leq E(z) \leq E_{1}=1.5\right)$. As can be seen, the dendritic $\langle 320\rangle$ specimen has most of its grains ranked in the first category (almost $75 \mathrm{pct}$ ), except for the first cross section $z=0$ where the grain size is only a few pixels in size and for the last one at $z=4 \mathrm{~cm}$ where few grains remain.

Turning our attention now to the seaweed grains selected at $1 \mathrm{~cm}$ (Figure 8(b)), many grains are definitely elongated. This elongation clearly appears in the statistics of the seaweed $\langle 210\rangle$ specimen shown in Figure 10(a); nearly half of the grains have an aspect ratio between 1.5 and 2 . The same was found for the seaweed $\langle 110\rangle$ specimen (i.e., $C_{\mathrm{Zn}}=55 \mathrm{wt}$ pct). Surprisingly, nearly the same proportion of elongated grains $1.5<E(z) \leq 2$ is found in each section, regardless of the height $z$. Keeping in mind that the section $z=0$ is already at some (small) distance from the nucleation surface due to polishing, this indicates that this specific grain-growth morphology occurs very early in the solidification process. Only the size of the grains changes as growth proceeds. Therefore, the average aspect ratio of the grains, $\bar{E}$, listed in Table II has been calculated for each specimen over all the sections analyzed.

The dendritic specimens have a value of $\bar{E}$ slightly larger than one, for several reasons: 


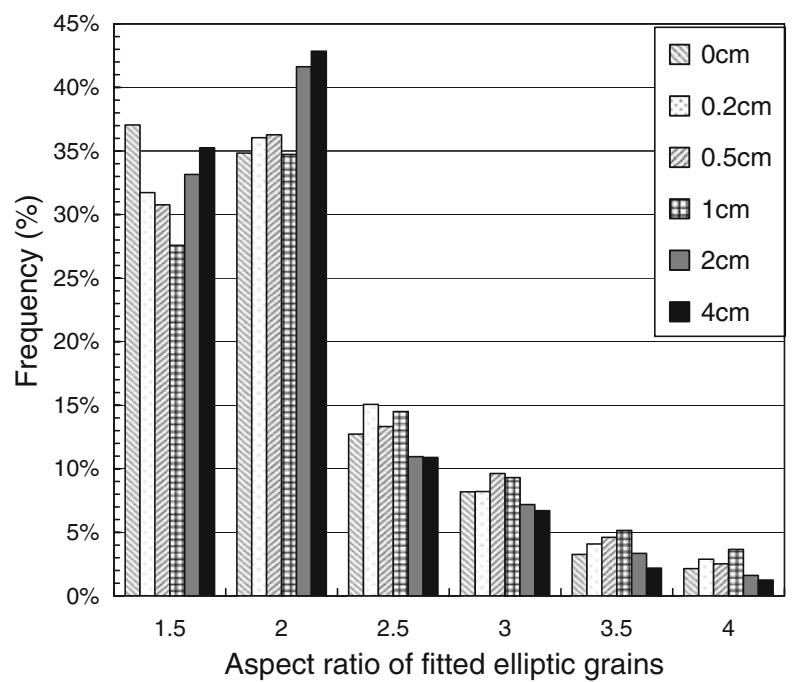

(a)

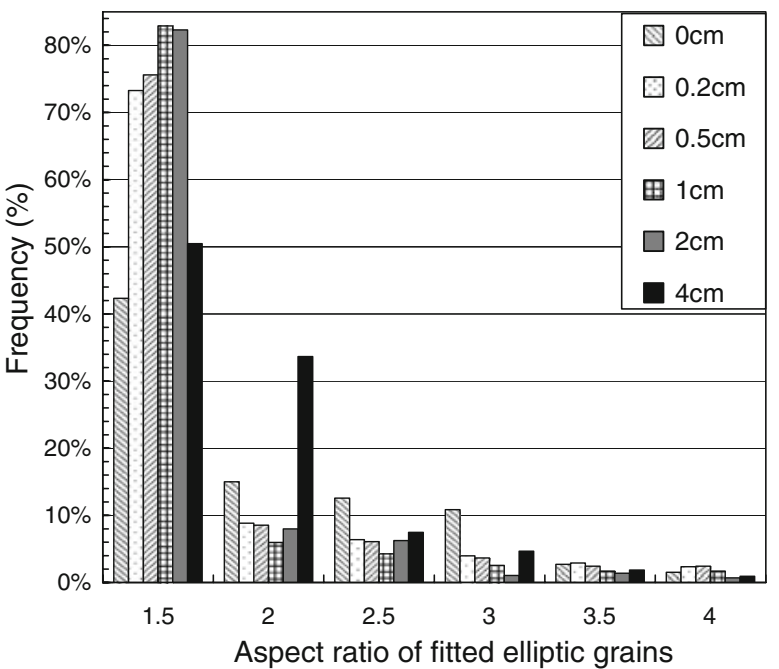

(b)

Fig. 10-Normalized aspect ratio distribution of grains seen in transverse sections of $(a)$ Al-35 wt pet $\mathrm{Zn}\langle 210\rangle$ seaweed specimen and (b) $\mathrm{Al}-50 \mathrm{wt}$ pct $\mathrm{Zn}\langle 320\rangle$ dendritic specimen. Gray levels and symbols correspond to various cross sections made at a distance $z$ from the surface of nucleation.

- The growth morphology might depend on the symmetry of dendrite side arms. The [100] dendritic growth is perfectly symmetric since four [010]$[0 \overline{1} 0]-[001]-[00 \overline{1}]$ side arms grow perpendicularly to the [100] trunk. However, if the trunk is misaligned with the thermal gradient, this already creates an anisotropic situation. During [110] dendritic growth, the situation is more complex as there are four $\langle 110\rangle$ side arms at 60 deg from the trunk direction, located in two (111) planes making an angle of $70.5 \mathrm{deg}$ With these four arms, the grains should be more elongated along the bisector plane. However, in the plane perpendicular to this bisector, two [110] and [110] arms are growing at $90 \mathrm{deg}$ from the trunk, thus making the situation more symmetric. The situation of $\langle 320\rangle$ dendritic growth is even more complicated since two $\langle 320\rangle$ arms are growing at $11 \mathrm{deg}$ from each $\langle 110\rangle$ direction. The average elongation factor $\bar{E}$ in this case starts to deviate from unity (Table II, $C_{\mathrm{Zn}}=50 \mathrm{wt}$ pct).

- Each grain shape results from the statistics of competition (and impingement) and is thus not necessarily symmetric.

- Finally, in the upper sections, the misorientation of the grains becomes comparable to the value of $5 \mathrm{deg}$ set to distinguish two different grains in the EBSD measurements. Setting a smaller value for the definition of grains does not make much sense, on the other hand, as subgrain misorientations on the order of a few degrees are anyway present in dendritic single-crystals. ${ }^{[15-17]}$ Therefore, in the upper transverse sections, one might sometimes look at clusters of grains slightly misaligned with respect to each other rather than individual grains.

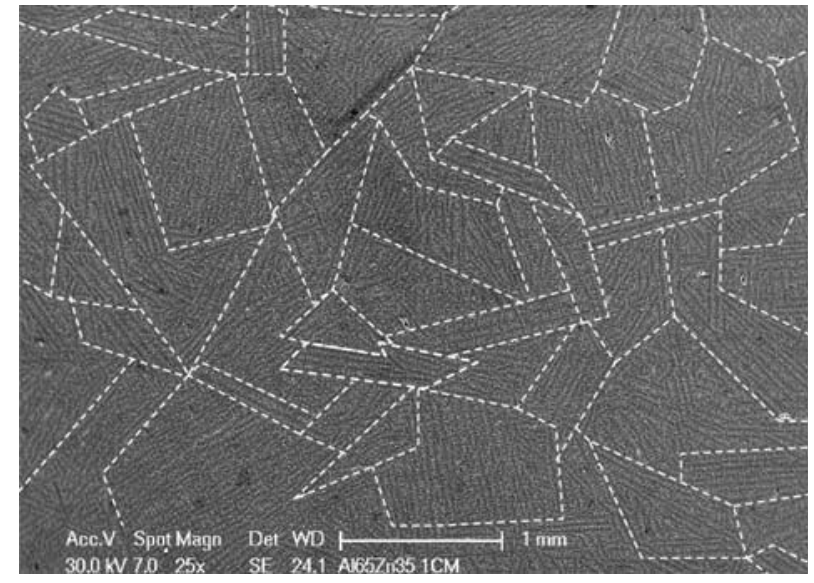

Fig. 11-SEM picture of a transverse section of Al-35 wt pet $\mathrm{Zn}$ at height $z=1 \mathrm{~cm}$. Grain boundaries are outlined with white dashed lines.

The elongation factor of the two seaweed specimens is definitely closer to two. Therefore, it must involve a quite different growth mechanism as compared with dendritic specimens. In order to see why grains are more elongated in the seaweed specimens, SEM was made on the transverse section $z=1 \mathrm{~cm}$ of the Al-35 wt pct $\mathrm{Zn}$ specimen (Figure 11). As can be seen on this picture using filtered secondary electrons, ${ }^{* *}$ each grain presents

**A bias voltage of $-300 \mathrm{~V}$ was used to detect secondary electrons with an Everton Thorenly detector. The contrast is then as for backscattered electrons, i.e., proportional to the $Z$-number of the elements.

some kind of a "layered" microsegregation contrast made of Al- and $\mathrm{Zn}$-rich regions. This shows that the 
seaweeds observed in longitudinal sections in Reference 1 are more or less growing in planes. These layers are very often parallel to the grain boundaries as a consequence of the growth mechanism. For both the Al-35 wt pct $\mathrm{Zn}$ and Al-55 wt pet $\mathrm{Zn}$ seaweed specimens, 4 or 5 elongated grains having their $\langle 210\rangle$, respectively $\langle 110\rangle$, growth direction perfectly aligned with the thermal gradient were selected. In each of them, the layers were clearly identified as corresponding to (001) planes. This is an indication that seaweeds are growing mostly in the forms of (001) layers; this growth mechanism could result from an interfacial energy $\gamma_{s l}$, which has a (001) inplane anisotropy fairly low compared to the out-of plane component.

\section{CONCLUSIONS}

In summary, the analysis of the texture evolution and grain density and morphology of DS Al-Zn specimens has revealed many important features:

- In dendritic specimens, the evolutions of the $\langle h k 0\rangle$ texture and grain density in transverse sections can depend on the initial grain density, the multiplicity of $\langle h k 0\rangle$ directions, the solute content (via the undercooling), etc. However, it is observed to occur within a characteristic distance of typically $2 \mathrm{~mm}$ in all three dendritic specimens $(\langle 110\rangle,\langle 320\rangle$, and $\langle 110\rangle)$. In the $\langle 210\rangle$ and $\langle 110\rangle$ seaweed specimens, the characteristic distance for these evolutions is nearly doubled.

- In dendritic specimens, the columnar grains appear fairly equiaxed in transverse sections, while they have an average elongation factor close to 2 in seaweed specimens. The elongation factor of seaweeds is linked to their growth morphology; they appear to grow as layered structures parallel to (001) planes.

From this analysis, it becomes evident that the case of textured 3-D seaweeds must be analyzed further. In particular, serial sectioning and 3-D reconstruction is being pursued in order to clearly visualize their growth morphology. On the other hand, direct measurement of the solid-liquid interfacial energy anisotropy is needed in order to do phase field simulations. At present, the dendrite orientation transition from $\langle 100\rangle$ to $\langle 110\rangle$ could be reproduced by phase field simulations ${ }^{[5]}$ using only the first- and second-order quadratic terms in the development of $\gamma_{s l}$. However, these simulations could only produce dendrites and not seaweeds and thus, a third-order term might be needed.

\section{ACKNOWLEDGMENTS}

The authors thank the Fonds Nationals Suisse de la recherche scientifique for its financial support (Grant No. 200021-105144) and the CIME (Interdisciplinary Centre for Electron Microscopy) of EPFL for providing the electron microscope facility. The authors acknowledge the valuable assistance of J.D Wagnière for DS experiments and helpful discussions with E. Boehm-Courjault about EBSD measurements.

\section{APPENDIX}

The probability $d p_{h k l}(\theta)$ of finding the thermal gradient $G$ at an angle $[\theta, \theta+d \theta]$ to a closest direction $[h k l]$ is given by the arc of circle shown in Figure 1 multiplied by $d \theta$ and divided by the surface $\pi / 12$ of the unit triangle. Therefore, one has

$$
p_{h k l}^{\prime}(\theta)=\frac{d p_{h k l}(\theta)}{d \theta}=\frac{12}{\pi} \sin \theta \int_{\phi_{d}} d \phi
$$

where $\phi_{d}$ represents the domain of the angles $\phi$ falling within the unit triangle and corresponding to the intersection with the unit sphere of the cone $\theta_{h k l}$ (continuous arc of circles shown in Figure 12). If this cone does not intersect the boundaries of the unit triangle, then the integral in Eq. [1] is simply $2 \pi$. If it does intersect one, two, or three boundaries limiting the unit triangle, then we have to calculate the values of $\phi$ corresponding to these intersections in order to find the total arc length within the unit triangle. Figure 1 shows the case where the cone $\theta_{h k l}$ intersects only the boundary connecting the [100] and [111] directions, while Figure 12 shows only the unit triangle in a stereographic projection and a cone $\theta_{h k l}$ giving the maximum number of intersections ( 2 with each boundary). The three planes limiting the unit triangle are given by (001) for the two directions [100] - [110], by (110) for the two directions [110] $-[111]$ and by $(011)$ for the two directions [100] - [111].

As the calculations give fairly complicated expressions, we just outline this derivation here. In the $\langle 100\rangle$ reference frame, we write the intersection $\mathbf{r}_{\cap}$ between the cone $\phi_{h k l}$ and one of the three boundaries as:

$$
\mathbf{r}_{\cap}=\left(\begin{array}{l}
\cos \alpha \\
\sin \alpha \cos \beta \\
\sin \alpha \sin \beta
\end{array}\right)
$$

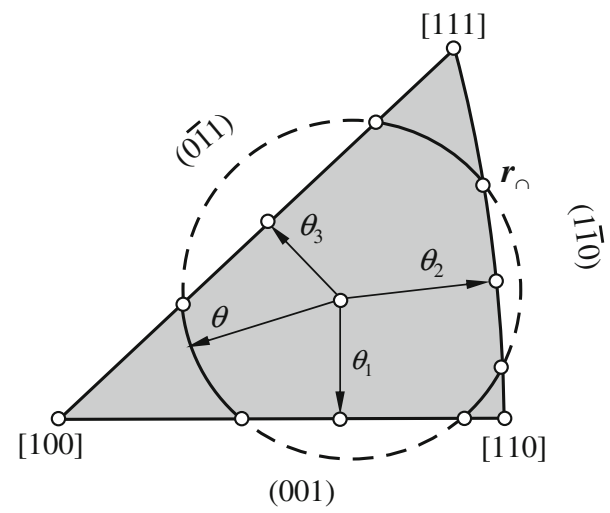

Fig. 12-Unit sphere triangle showing the intersections of the cone $\theta_{h k l}$ with the three boundaries and the three characteristic values $\theta_{1}$, $\theta_{2}$, and $\theta_{3}$ of the cones when they just touch the boundaries. 
where $\alpha$ is the angle between $\mathbf{r}_{\cap}$ and [100], and $\beta$ the angle between $\mathbf{r}_{\cap}$ and the (001) plane. In order to detect whether there is an intersection, we first identify the three angles $\theta$ for which the corresponding cone $\theta_{h k l}$ just touches one of the three boundaries (Figure 12). Using simple geometric considerations and normalizing the direction $[h k l]$ to a unit vector, i.e., $h^{2}+k^{2}+l^{2}=1$, it is straightforward to show that

$$
\begin{gathered}
\text { Plane (001): } \quad \theta_{1}=\arccos \sqrt{h^{2}+k^{2}} \\
\text { Plane (110): } \quad \theta_{2}=\arccos \sqrt{\frac{(h+k)^{2}}{2}+l^{2}} \\
\text { Plane }(0 \overline{1} 1): \quad \theta_{3}=\arccos \sqrt{h^{2}+\frac{(k+l)^{2}}{2}}
\end{gathered}
$$

If $\theta<\min \left(\theta_{1}, \theta_{2}, \theta_{3}\right)$, there is not intersection with the boundaries and the integral of Eq. [1] is simply equal to $2 \pi$. As the angle $\theta$ is increased, the intersections with the various boundaries are calculated. If they exist, they are given by

Plane (001): $\quad \beta=0$

$$
\alpha=\arccos \left[\frac{h \cos \theta \pm k \sqrt{h^{2}+k^{2}-\cos ^{2} \theta}}{h^{2}+k^{2}}\right]
$$

Plane $(1 \overline{1} 0)$ :

$\beta=\arccos \frac{1}{\tan \alpha}$

$\alpha=\arccos \left[\frac{(h+k) \cos \theta \pm l \sqrt{(h+k)^{2}+2\left(l^{2}-\cos ^{2} \theta\right)}}{(h+k)^{2}+2 l^{2}}\right]$
Using Eqs. [6] through [8] giving the angles $\alpha$ and $\beta$ of the intersection points $\mathbf{r}_{\cap}$ in the $\langle 100\rangle$ reference system, the new spherical coordinates can be found in a system accepting the direction $[h k l]$ as the $z$ axis. One retrieves of course the angle $\theta$ of the cone for the angle between $\mathbf{r}_{\cap}$ and the $z$ axis. The azimuthal angle $\phi_{\cap}$ of the intersection points being found out in the new reference system, the total arc length(s) intervening in Eq. [1] is obtained, and thus the distribution $p_{h k l}^{\prime}(\theta)$. These distributions are directly plotted in the various experimental results showing the statistics of the orientations measured by EBSD.

\section{REFERENCES}

1. F. Gonzales and M. Rappaz: Metall. Mater. Trans. A, 2006, vol. 37A, pp. 2797-806.

2. W. Kurz and D. Fisher: Fundamentals of Solidification, Trans Tech Publications, Aedermansdorf, Switzerland, 1998.

3. S. Akamatsu, G. Faivre, and T. Ihle: Phys. Rev., 1995, vol. E51, pp. 4751-73.

4. S-K. Chan, H.H. Reimer, and M. Kahlweit: J. Cryst. Growth, 1976, vol. 32, pp. 303-15.

5. T. Haxhimali, A. Karma, F. Gonzales, and M. Rappaz: Nature Mater., 2006, vol. 5, pp. 660-64.

6. S. Liu, R.E. Napolitano, and R. Trivedi: Acta Mater., 2001, vol. 49 , p. 4271.

7. R.E. Napolitano and S. Liu: Phys. Rev., 2004, vol. B70, p. 214103.

8. A. Passerone and N. Eustathopoulos: Acta Mater., 1982, vol. 30, p. 1349.

9. Binary Alloy Phase Diagrams, T. Massalski, ed., ASM, Metals Park OH, 1986 p. 1.

10. B. Chalmers: Principles of Solidification, Wiley, New York, NY, 1964.

11. H. Esaka, J. Stramke, and W. Kurz: Columnar Dendrite Growth in Succinonitrile-Acetone Alloys, Film, Ecole Polytechnique Fédérale de Lausanne, LSMX, Lausanne, Switzerland, 1985.

12. C.-A. Gandin, M. Rappaz, D. West, and B.L. Adams: Metall. Trans. A, 1995, vol. 25A, pp. 1543-51.

13. D.W. Hoffman and J.W. Cahn: Surf. Sci., 1972, vol. 31, pp. 36888.

14. C. Herring: Phys. Rev., 1951, vol. 82, p. 87.

15. A. Sémoroz, Y. Durandet, and M. Rappaz: Acta Mater., 2001, vol. 49, pp. 529-41.

16. M. Rappaz and E. Blank: J. Cryst. Growth, 1986, vol. 74, pp. 6776.

17. N. Siredey, M. Boufoussi, S. Denis, and J. Lacaze: J. Cryst. Growth, 1993, vol. 130, p. 132.

Plane $(0 \overline{1} 1)$ :

$\beta=\frac{\pi}{4}$

$\alpha=\arccos \left[\frac{2 h \cos \theta \pm(k+l) \sqrt{(k+l)^{2}+2\left(h^{2}-\cos ^{2} \theta\right)}}{2 h^{2}+(k+l)^{2}}\right]$ 